\title{
Effects of Hyperbaric Oxygen Therapy on Inflammation, Oxidative/Antioxidant Balance, and Muscle Damage after Acute Exercise in Normobaric, Normoxic and Hypobaric, Hypoxic Environments: A Pilot Study
}

\author{
Jinhee Woo $^{1,+}$, Jae-Hee Min ${ }^{1,+}$, Yul-Hyo Lee ${ }^{2}$ and Hee-Tae Roh ${ }^{1, *(D)}$ \\ 1 Department of Physical Education, College of Arts and Physical Education, Dong-A University, \\ Busan 49315, Korea; sports@dau.ac.kr (J.W.); jop9508@naver.com (J.-H.M.) \\ 2 Department of Taekwondo, Youngsan University, Yangsan-si 50510, Korea; health@ysu.ac.kr \\ * Correspondence: dau0409@dau.ac.kr; Tel.: +82-51-200-7835; Fax: +82-51-200-7805 \\ + The first two authors contributed equally to this work.
}

Received: 14 September 2020; Accepted: 7 October 2020; Published: 10 October 2020

check for updates

\begin{abstract}
The purpose of this study was to investigate the effects of hyperbaric oxygen therapy (HBOT) on inflammation, the oxidative/antioxidant balance, and muscle damage after acute exercise in normobaric, normoxic $(\mathrm{NN})$ and hypobaric, hypoxic $(\mathrm{HH})$ environments. Eighteen healthy males were selected and randomly assigned to three groups: exercise in NN conditions (NN group, $n=6$ ), HBOT treatment after exercise in NN conditions (HNN group, $n=6$ ), and HBOT treatment after exercise in $\mathrm{HH}$ conditions (HHH group, $n=6$ ). All subjects performed treadmill running for $60 \mathrm{~min}$ at $75-80 \%$ maximum heart rate (HRmax) exercise intensity under each condition. The HBOT treatments consisted of breathing $100 \%$ oxygen at 2.5 atmosphere absolute (ATA) for $60 \mathrm{~min}$. Blood samples were collected before exercise (BE), after exercise (AE), and after HBOT (AH) to examine inflammation (fibrinogen, interleukin-6 [IL-6], and tumor necrosis factor- $\alpha$ (TNF- $\alpha)$ ), the oxidative/antioxidant balance (derivatives of reactive oxygen metabolites (d-ROMs) and the biological antioxidant potential $(\mathrm{BAP})$ ), and muscle damage (creatine kinase (CK) and lactate dehydrogenase (LDH)). Plasma fibrinogen, serum IL-6, CK, and LDH levels were significantly increased AE compared to BE in all groups $(p<0.05)$. Plasma fibrinogen levels were significantly decreased AH compared to AE in all groups $(p<0.05)$, and the HNN group had a significantly lower AH compared to BE $(p<0.05)$. Serum IL-6 levels were significantly decreased AH compared to AE in the HNN and HHH groups $(p<0.05)$. Serum CK levels were significantly decreased AH compared to AE in the HHH group $(p<0.05)$. Serum LDH levels were significantly decreased AH compared to AE in the HNN and HHH groups $(p<0.05)$, and the NN and HNN groups had significantly higher AH serum LDH levels compared to $\mathrm{BE}(p<0.05)$. These results suggest that acute exercise in both the NN and $\mathrm{HH}$ environments could induce temporary inflammatory responses and muscle damage, whereas HBOT treatment may be effective in alleviating exercise-induced inflammatory responses and muscle damage.
\end{abstract}

Keywords: healthy young individuals; acute exercise; inflammation; oxidative stress; muscle damage; hypoxia; hyperbaric oxygen therapy

\section{Introduction}

Regular exercise training is not only effective in preventing and relieving the symptoms of metabolic diseases, such as hypertension, hyperlipidemia, and diabetes mellitus, and cardiovascular 
disease by reducing the levels of chronic inflammation and oxidative stress (OS) within the body, but it can also induce numerous other health benefits by increasing skeletal muscle mass [1,2]. In contrast, acute exercise, which stresses body tissues, can cause excessive OS accompanied by muscle damage [3,4]. The body exhibits decreased muscular function and/or inflammatory response due to excessive muscle fatigue without proper recovery from the damage, and this can lead to exercise impairment as well as a reduction in the ability to perform exercise $[3,5]$. Previous studies suggested that inflammatory cytokines, such as interleukin-6 (IL-6) and tumor necrosis factor- $\alpha$ (TNF- $\alpha$ ), and fibrinogen could be blood biomarkers for the inflammatory response induced by exercise [6]. Serum creatine kinase (CK) and lactate dehydrogenase (LDH) levels are typical indices that reflect exercise-induced muscle damage [7]. In addition, exercising in hypoxic conditions can put additional stress on the body compared to exercising in normoxia [8]. Hypoxia was reported to increase the generation of reactive oxygen species, have negative impacts on antioxidant defenses, and cause severe OS and inflammation through TNF- $\alpha$ release and nuclear factor- $\mathrm{kB}$ (NF- $\mathrm{kB}$ ) activation $[9,10]$.

Strategies, such as consuming food (nutrients) with antioxidant and anti-inflammatory effects [11,12], low-level laser therapy [13], and cold water immersion [14] treatment, have been suggested to reduce the inflammatory response and excessive OS generation from acute exercise, and help exercise-induced damaged muscles recover faster. The strategies remain controversial, but some of them were proven to be effective. Recently, studies reported that hyperbaric oxygen therapy (HBOT) could be effective in early recovery from exercise-related muscular injury due to its beneficial effect on cell recovery and tissue repair $[15,16]$. HBOT is a treatment procedure where the patient is intermittently given $100 \%$ pure oxygen under pressure higher than atmospheric pressure ( 1 atmosphere absolute [ATA] $=101 \mathrm{kPa}$ ) in a hyperbaric chamber. The typical range of pressure is 2-2.8 ATA and it is effective at 1.4 ATA or greater pressure [15]. HBOT is a treatment that can be applied to various conditions, including carbon monoxide poisoning, compromised skin graft and flaps, crushing injuries, necrotizing soft tissue infections, and non-healing ulcers with arterial inefficiencies. Moghadam et al. [15] suggested this as a possible treatment for sports musculoskeletal injuries as it can enhance oxygen delivery, reduce edema and pathologic inflammation, and mitigate ischemia/reperfusion injury. Chen et al. [16] applied HBOT treatment to 41 athletes with exercise-related muscle damage and reported that muscle damage indices like $\mathrm{CK}$, glutamic oxaloacetate transaminase (GOT), and myoglobin were significantly decreased.

However, previous studies $[17,18]$ reported that HBOT treatment in the recovery phase after exercise had no significant effect in relieving exercise-induced muscle soreness and damage. Despite the experimental evidence [19] showing that HBOT treatment could mitigate OS in human endothelial cells, only a few studies have verified the effect of HBOT treatment on exercise-induced inflammation and OS in the recovery phase after exercise. Moreover, the effect of HBOT treatment after exercising in hypoxic conditions which can induce higher level of OS and more inflammatory response was not reported. The purpose of this study was to verify the levels of inflammation, OS, and muscle damage according to normobaric, normoxic and hypobaric, hypoxic exercise environments, and investigate the beneficial effect of HBOT treatment in relieving inflammation, OS, and muscle damage in the recovery phase after acute exercise.

\section{Methods}

\subsection{Subjects}

The prospective number of subjects was calculated to be 18 subjects for an effect size of 0.40 , an $\alpha$ value of 0.05 , and a desired statistical power $(1-\beta)$ of 0.80 . We posted a recruitment notice at Dong-A University for three weeks from 1 December 2018, and recruited 18 healthy males with normal body composition (body mass index (BMI) under $30 \mathrm{~kg} / \mathrm{m}^{2}$ ) with no musculoskeletal disorders and no surgical experience related to that in the past one year. The 18 selected subjects were randomly assigned to three groups of six people each: one exercised in normobaric, normoxic (NN) conditions (NN group), one had 
HBOT treatment after exercising in NN conditions (HNN group), and the last one had HBOT treatment after exercising in hypobaric, hypoxic (HH) conditions (HHH group). Approval for this study was obtained from the Ethics Committee of Dong-A University (ID: 2-1040709-AB-N-01-201810-HR-032-04). All subjects were fully informed of the study procedures and signed an informed consent form indicating that they understood the study procedures and the risks and benefits of participation. The characteristics of the subjects at baseline are shown in Table 1 . There were no significant differences between the groups.

Table 1. Characteristics of the subjects at baseline.

\begin{tabular}{ccccc}
\hline Variable/Group & NN $(\boldsymbol{n}=\mathbf{6})$ & HNN $(\boldsymbol{n}=\mathbf{6})$ & HHH $(\boldsymbol{n}=\mathbf{6})$ & $\boldsymbol{p}_{\text {Value }}^{\text {\& }}$ \\
\hline Age $(\mathrm{years})$ & $23.67 \pm 3.44$ & $21.67 \pm 2.34$ & $23.00 \pm 2.76$ & 0.490 \\
Height $(\mathrm{cm})$ & $174.33 \pm 2.89$ & $173.87 \pm 4.30$ & $176.92 \pm 5.55$ & 0.450 \\
Weight $(\mathrm{kg})$ & $75.63 \pm 4.80$ & $72.95 \pm 5.23$ & $74.43 \pm 6.03$ & 0.694 \\
BMI $\left(\mathrm{kg} / \mathrm{m}^{2}\right)$ & $24.88 \pm 1.53$ & $24.12 \pm 1.29$ & $23.75 \pm 0.68$ & 0.290 \\
Body fat $(\%)$ & $22.28 \pm 7.35$ & $21.20 \pm 5.23$ & $20.97 \pm 3.08$ & 0.907 \\
HRrest $($ beats/min) & $73.33 \pm 8.82$ & $70.33 \pm 10.75$ & $66.00 \pm 6.87$ & 0.385 \\
HRmax (beats/min) & $196.33 \pm 3.44$ & $198.33 \pm 2.34$ & $197.00 \pm 2.76$ & 0.490 \\
$\mathrm{VO}_{2}$ max $(\mathrm{mL} / \mathrm{kg} / \mathrm{min})$ & $47.17 \pm 2.30$ & $49.15 \pm 2.82$ & $48.29 \pm 0.87$ & 0.308 \\
Fibrinogen $(\mathrm{mg} / \mathrm{dL})$ & $230.00 \pm 24.92$ & $231.67 \pm 33.68$ & $221.83 \pm 39.63$ & 0.862 \\
IL-6 $(\mathrm{pg} / \mathrm{mL})$ & $909.17 \pm 136.62$ & $924.47 \pm 125.85$ & $912.83 \pm 48.01$ & 0.969 \\
TNF- $\alpha(\mathrm{pg} / \mathrm{mL})$ & $938.80 \pm 8.52$ & $975.46 \pm 34.59$ & $971.19 \pm 51.42$ & 0.192 \\
d-ROMs $(\mathrm{CARR} . \mathrm{U})$ & $289.67 \pm 34.07$ & $267.83 \pm 44.21$ & $274.67 \pm 50.54$ & 0.680 \\
BAP $(\mu \mathrm{\mu mol} / \mathrm{L})$ & $2726.67 \pm 220.81$ & $2583.67 \pm 329.77$ & $2632.50 \pm 186.14$ & 0.619 \\
CK $(\mathrm{U} / \mathrm{L})$ & $205.85 \pm 44.02$ & $221.58 \pm 38.27$ & $218.50 \pm 64.36$ & 0.849 \\
LDH $(\mathrm{U} / \mathrm{L})$ & $312.83 \pm 42.98$ & $335.67 \pm 45.91$ & $291.50 \pm 39.67$ & 0.237 \\
\hline
\end{tabular}

Values are mean \pm standard deviation. NN: exercised in normobaric, normoxic conditions; HNN: HBOT treatment after exercising in normobaric, normoxic conditions; HHH: HBOT treatment after exercising in in hypobaric, hypoxic conditions; HRrest: heart rate at rest, $\mathrm{HRmax}$ : heart rate at peak exercise; $\mathrm{VO}_{2}$ max: maximal oxygen uptake; IL-6: interleukin-6; TNF- $\alpha$ : tumor necrosis factor- $\alpha$; d-ROMs: derivatives of reactive oxygen metabolites; BAP: biological antioxidant potential; CK: creatine kinase; LDH: lactate dehydrogenase; ${ }^{*}$ Determined using the one-way ANOVA.

\subsection{Anthropometric Measures and $\mathrm{VO}_{2}$ max Test}

Anthropometric measurements, which were taken one week before beginning the main exercise test (NN and $\mathrm{HH}$ conditions), included height, body composition, and maximal oxygen uptake ( $\left.\mathrm{VO}_{2} \mathrm{max}\right)$. Height and body composition were measured using a multi-frequency bioelectrical impedance analyzer (Accuniq BC720; SELVAS Healthcare, Daejeon, Korea). $\mathrm{VO}_{2}$ max was measured on a treadmill (T150; Cosmed, Rome, Italy) according to the Bruce protocol [20], using a gas analyzer (Quark CPET; Cosmed, Rome, Italy) based on the breath-by-breath method.

\subsection{Exercise Environment Setting and HBOT Procedure Protocol}

In the exercise environment setting and for HBOT treatment, a multi-pressure chamber (Interocean I.O medical, Busan, Korea) was used that could reflect the atmospheric pressure and create conditions of hypobaric/hypoxia and hyperbaric/hyperoxia. More specifically, the subjects in each group (NN, $\mathrm{HNN}$, and $\mathrm{HHH}$ ) ran on a treadmill for 60 min at 75-80\% HRmax intensity in NN conditions (760 Torr, approximately $20.9 \%$ oxygen) for those at sea level and $\mathrm{HH}$ conditions (526 Torr, approx. $14.5 \%$ oxygen) for those at an altitude of $3000 \mathrm{~m}$. Each treadmill run was initiated with the Bruce protocol [20] while wearing a Bluetooth heart rate sensor (H10; Polar Electro Oy, Kempele, Finland). When the subject's heart rate was monitored and the target heart rate reached, the treadmill slope was fixed at $0 \%$, and the treadmill speed was adjusted to control the exercise intensity. For HBOT treatment of the HNN and HHH groups, the protocol of inhaling 100\% oxygen at an increased air pressure of 2.5 ATA was used according to a previous study by Shimoda et al. [21]. The subjects were compressed to 2.5 ATA over $15 \mathrm{~min}$, after which they breathed $100 \%$ oxygen for 60 min delivered through a tight-fitting mask covering the nose and mouth for three 20 -min periods with 5-min breaks in ambient air (20.9\% oxygen). 
Two 5-min air breaks in ambient air were carried out in 2.5 ATA. At the end of the 60-min period, $15 \mathrm{~min}$ was allowed for the participant to decompress to atmospheric pressure. The treatment method for this study requires a 5-min air break for every 20-min oxygen inhalation to avoid oxygen toxicity. The treatment was applied for $105 \mathrm{~min}$ including $45 \mathrm{~min}$ of air breaks (Figure 1).

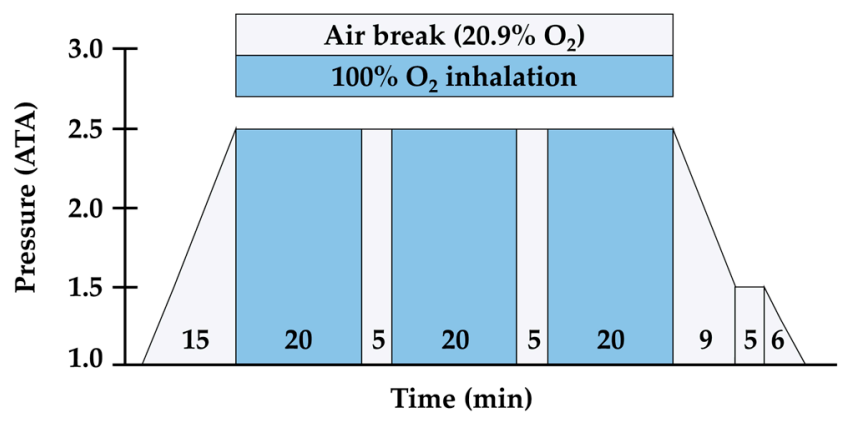

Figure 1. Hyperbaric oxygen therapy (HBOT) treatment protocol.

\subsection{Blood Collection and Analyses}

Using a 22-gauge needle, $10 \mathrm{~mL}$ of blood was collected from the antecubital vein of each subject before exercise (BE), after exercise (AE), and after HBOT (AH) into a serum separator tube, an ethylenediamine tetraacetate-containing tube, and a sodium citrate-containing tube (all tubes were purchased from Becton Dickinson, Franklin Lakes, NJ, USA). The collected blood samples were centrifuged for $15 \mathrm{~min}$ at $3000 \mathrm{rpm}$ and were then stored at $-80^{\circ} \mathrm{C}$ until analysis. The analysis of plasma fibrinogen levels was performed using a chronometric assay (Fibriprest, Diagnostica Stago, Asnieres, France). Serum IL-6 and TNF- $\alpha$ levels were measured using a human IL-6 Duoset enzyme-linked immunosorbent assay (ELISA) kit (DY206; R\&D Systems, Minneapolis, MN, USA) and a human TNF-alpha Quantikine HS ELISA kit (HSTA00E; R\&D Systems, Minneapolis, MN, USA), respectively. A microplate reader (Sunrise ${ }^{\text {TM}}$; TECAN Austria GMBH, Grödig/Salzburg, Austria) was used to measure the absorbance at $450 \mathrm{~nm}$ for quantification. The analyses of serum derivatives of reactive oxygen metabolites (d-ROMs) and the biological antioxidant potential (BAP) levels were measured using a commercial assay kit (Diacron SRL, Proma, Italy) as described by Hussein et al. [22]. Serum $\mathrm{CK}$ and LDH levels were measured at $680 \mathrm{~nm}$ absorbance using a standard microwell plate reader (Molecular Devices, Orleans, CA, USA).

\subsection{Statistical Analyses}

Statistical analyses were performed with SPSS version 25.0 for Windows (SPSS Inc., Chicago, IL, USA). The data are presented as the mean \pm standard deviation. A two-way repeated analysis of variance (ANOVA) was performed to identify the differences in normally distributed data. Tests of normality for all measured values were conducted using the one-sample Kolmogorov-Smirnov test. When significant group by time interactions occurred, the simple main effects were assessed using a one-way ANOVA. The Tukey post hoc test was used as a conservative locator of significant differences. Statistical significance was set at $p<0.05$.

\section{Results}

\subsection{Changes in Variables Related to Inflammation}

The changes in inflammatory markers according to HBOT after exercise in the $\mathrm{NN}$ and $\mathrm{HH}$ environments are shown in Figure 2. The two-way repeated ANOVA demonstrated a significant difference in the group by time interaction for plasma fibrinogen $(F=3.356, p=0.022)$ and serum IL-6 $(F=13.059, p<0.001)$ levels. Plasma fibrinogen levels were significantly increased AE compared to $\mathrm{BE}$ in all groups, and all groups were significantly decreased AH $(p<0.05)$. In addition, the HNN 
group had significantly lower AH plasma fibrinogen levels compared to BE $(p<0.05)$. Serum IL-6 levels were significantly increased AE compared to BE in all groups, and the levels in the HNN and $\mathrm{HHH}$ groups were significantly decreased AH $(p<0.05)$. In contrast, the serum TNF- $\alpha(F=1.131$, $p=0.361$ ) levels were not significantly different between any groups or time points.
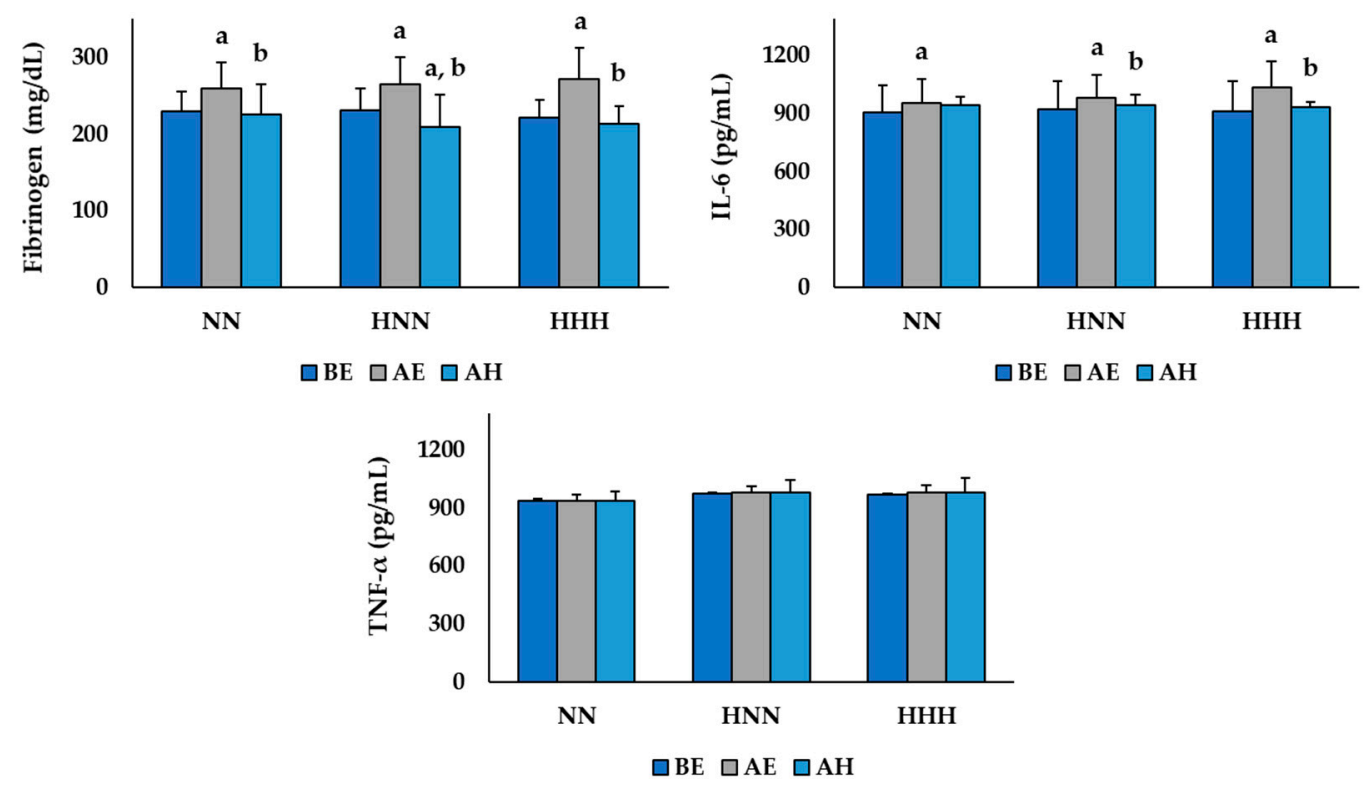

Figure 2. Changes in inflammatory markers according to HBOT after exercise in NN and $\mathrm{HH}$ environments. Values are mean \pm standard deviation; $\mathrm{NN}$ : exercised in normobaric, normoxic conditions; HNN: HBOT treatment after exercising in normobaric, normoxic conditions; $\mathrm{HHH}$ : HBOT treatment after exercising in in hypobaric, hypoxic conditions; BE: before exercise; AE: after exercise; AH: after HBOT; IL-6: interleukin-6; TNF- $\alpha$ : tumor necrosis factor- $\alpha$; ${ }^{\text {a }}$ Significant difference with BE $(p<0.05){ }^{\text {b }}$ Significant difference with $\operatorname{AE}(p<0.05)$.

\subsection{Changes in Variables Related to Oxidative/Antioxidant Balance}

The changes in oxidative/antioxidant balance markers according to HBOT after exercise in the $\mathrm{NN}$ and $\mathrm{HH}$ environments are shown in Figure 3. The two-way repeated ANOVA demonstrated no significant difference in the group by time interaction for serum d-ROMs $(F=0.512, p=0.728)$ and $\operatorname{BAP}(F=0.657, p=0.626)$ levels.
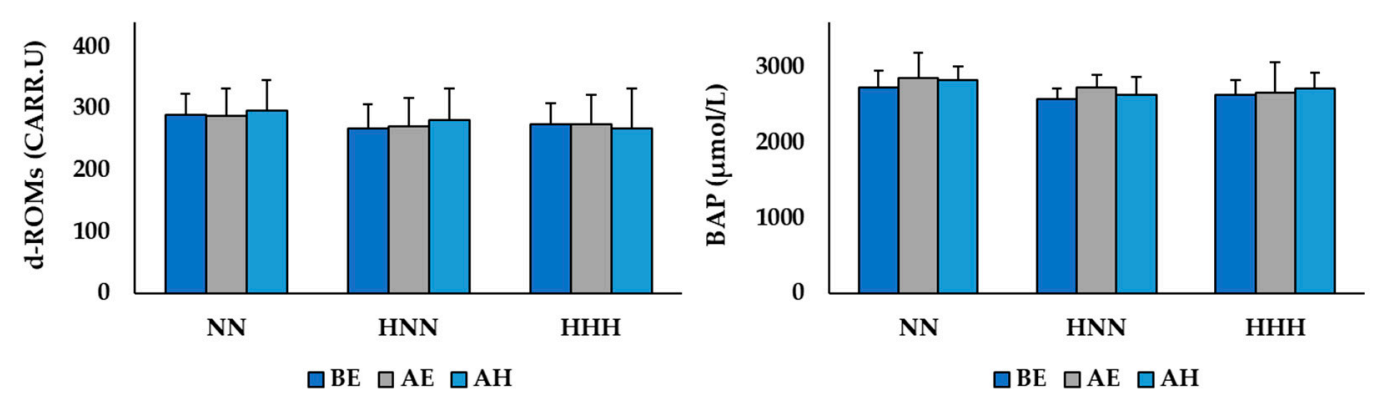

Figure 3. Changes in oxidative/antioxidant balance markers according to HBOT after exercise in $\mathrm{NN}$ and $\mathrm{HH}$ environments. Values are mean \pm standard deviation; NN: exercised in normobaric, normoxic conditions; HNN: HBOT treatment after exercising in normobaric, normoxic conditions; HHH: HBOT treatment after exercising in in hypobaric, hypoxic conditions; BE: before exercise; AE: after exercise; $\mathrm{AH}$ : after HBOT d-ROMs: derivatives of reactive oxygen metabolites; BAP: biological antioxidant potential. 


\subsection{Changes in Variables Related to Muscle Damage}

The changes in muscle damage markers according to HBOT after exercise in the $\mathrm{NN}$ and $\mathrm{HH}$ environments are shown in Figure 4. The two-way repeated ANOVA demonstrated a significant difference in the group by time interaction for serum CK $(F=6.880, p<0.001)$ and LDH $(F=10.362$, $p<0.001)$ levels. Serum CK levels were significantly increased AE compared to BE in all groups, and the level in the HHH group was significantly decreased AH $(p<0.05)$. Serum LDH levels were significantly increased AE compared to BE in all groups, and the levels in the HNN and HHH groups were significantly decreased $\mathrm{AH}(p<0.05)$. In addition, the $\mathrm{NN}$ and $\mathrm{HNN}$ groups had significantly higher serum LDH levels AH compared to BE $(p<0.05)$.
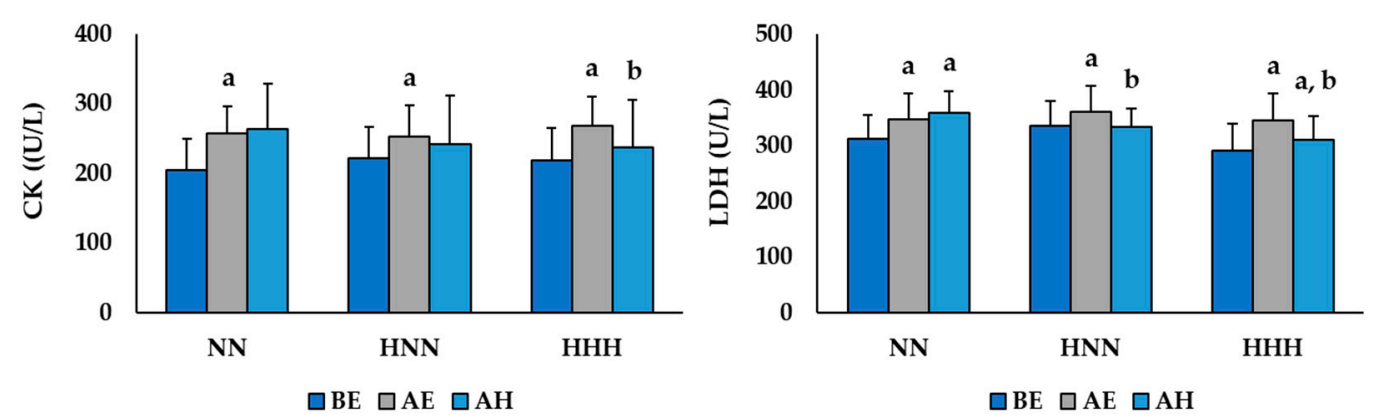

Figure 4. Changes muscle damage markers according to HBOT after exercise in $\mathrm{NN}$ and $\mathrm{HH}$ environments. Values are mean \pm standard deviation; $\mathrm{NN}$ : exercised in normobaric, normoxic conditions; HNN: HBOT treatment after exercising in normobaric, normoxic conditions; HHH: HBOT treatment after exercising in in hypobaric, hypoxic conditions; BE: before exercise; AE: after exercise; AH: after HBOT; CK: creatine kinase; LDH: lactate dehydrogenase; ${ }^{a}$ Significant difference with $\mathrm{BE}(p<0.05)$; b Significant difference with $\mathrm{AE}(p<0.05)$.

\section{Discussion}

Inflammation is a reaction that causes fever and pain in the body in response to foreign antigens and tissue damage, such as in skeletal muscles. Even though it is a necessary defensive reaction that helps damaged tissue to recover, it can also cause other diseases like cardiovascular disease [23] and is closely related to musculoskeletal damage and muscle fatigue [24,25]. In some previous studies [6,26], the levels of fibrinogen, IL-6, and TNF- $\alpha$ in the blood have been reported to be indices reflecting the inflammatory response induced by acute exercise. This study analyzed the levels of plasma fibrinogen, serum IL-6, and TNF- $\alpha$ to verify changes in the inflammatory response depending upon the exercise performed in $\mathrm{NN}$ and $\mathrm{HH}$ environments and investigated the effect of HBOT on recovery. The results of this study showed that the levels of plasma fibrinogen and serum IL-6 significantly increased after exercise in all groups, but there was no significant difference between the exercise environments (NN vs. $\mathrm{HH}$ ). These results are consistent with previous studies $[6,27]$ that demonstrated that acute exercise significantly increased the levels of fibrinogen and IL-6 in the blood by causing an inflammatory response, and suggest that exercising in an $\mathrm{HH}$ environment, which corresponds to conditions at an altitude of about $3000 \mathrm{~m}$, did not lead to additional inflammatory responses compared to exercising in an NN environment. Liakos et al. [6] reported that the levels of fibrinogen and IL-6 were significantly increased after maximal treadmill exercise testing using the Bruce protocol, and in Stelzer et al.'s study [27], the levels of fibrinogen and IL-6 were analyzed to verify changes in the inflammatory response with ultra-endurance exercise. The results showed that the fibrinogen and IL-6 levels were significantly increased after the race. Furthermore, Kasai et al. [26] conducted a repeated sprint exercise with 10 male athletes in hypoxic (fraction of inspired oxygen $\left(\mathrm{F}_{\mathrm{i}} \mathrm{O}_{2}=14.5 \%\right.$, equivalent to a simulated altitude of $3000 \mathrm{~m})$ and normoxic $\left(\mathrm{F}_{\mathrm{i}} \mathrm{O}_{2}=20.9 \%\right)$ conditions. Although the levels of IL-6 increased significantly after exercise in both conditions, there was no significant difference in levels between the two environmental conditions, which supports the results of this study. Additionally, 
the plasma fibrinogen levels were significantly lower at the BE point than at the AH point in the HNN group in this study. Additionally, the level of serum IL-6 decreased significantly at the AH point compared to the $\mathrm{AE}$ point in both the $\mathrm{HNN}$ and $\mathrm{HHH}$ groups, but there was no significant decrease in the $\mathrm{NN}$ group. This implies that HBOT treatment after exercise can be effective in relieving exercise-induced inflammation, supporting the findings of previous studies [28] that HBOT treatment could help athletics recover while training due to its effect to relieve inflammation.

Oxygen is a crucial factor in generating energy (adenosine triphosphate), which is required by the body for use in various metabolic processes when the body is resting and also when it is exercising and recovering. However, the incomplete reduction of oxygen during metabolic processes produces reactive oxygen species (ROS) that can be toxic to the body. Furthermore, the levels of OS in the body increase due to the accelerated production of ROS during highly intensive exercise, which requires a greater oxygen supply [3]. This study analyzed the levels of serum d-ROMs and BAP to verify the oxidative/antioxidant balance after exercising in $\mathrm{NN}$ and $\mathrm{HH}$ environments and investigated the effect of HBOT treatment on recovery. The levels of serum d-ROMs and BAP were used as indices of exercise-induced oxidative stress in a number of previous studies [29-31] as they reflect the level of OS and antioxidant capacity, respectively. Specifically, Sugita et al. [29] and Martarelli et al. [30] reported that the levels of d-ROMs and BAP increased significantly after incremental cycle exercise and mountain bike exercise in separate studies, and the levels of d-ROMs and BAP increased significantly, and the levels were also increased after cycling in $75 \% \mathrm{VO}_{2}$ max intensity in Aoki et al.'s study [31]. However, in this study, no statistically significant difference was found in either the d-ROMs or BAP levels. It is considered that high antioxidant activity and high levels of maximal aerobic power $\left(\mathrm{VO}_{2} \mathrm{max}\right)$ of the participants in this study played major roles in the study results. That is, OS production in the body caused from an imbalance between pro-oxidants and antioxidants, such as excessive ROS production and/or compromised intrinsic antioxidant defense [32], may have contributed to the higher resting level serum BAP of the participants in this study at $2647.61 \pm 245.45 \mu \mathrm{mol} / \mathrm{L}$ than the resting level BAP (from 1938.5 to $2347.3 \mu \mathrm{mol} / \mathrm{L}$ ) in previous studies [29-31], which proved that the d-ROMs and BAP levels increased after acute exercise. Additionally, the $\mathrm{VO}_{2}$ max level of the participants in this study was $48.20 \pm 2.19 \mathrm{~mL} / \mathrm{kg} / \mathrm{min}$ which is above the 60th percentile for adult men in their 20s [33]. Bachi et al. [34] suggested that the level of OS after exercise could be lower in people with higher $\mathrm{VO}_{2}$ max and total antioxidant activity. However, considering that high-intensity acute exercise can be one of the main causes of increased OS in the body, it seems necessary to verify this in additional studies by analyzing serum ROS levels and/or antioxidant enzymes such as superoxide dismutase (SOD) and catalase, not only d-ROMs and BAP levels.

As exercise-induced muscle damage is caused by acute or long-term damage to muscle cells and tissues, it is possible to indirectly estimate the level of damage from the levels of enzymes like CK and $\mathrm{LDH}$ that are released into the blood from muscle tissues during exercise $[7,15]$. Serum muscle enzyme levels increase not only in the body of athletes who exercise intensively but also in ordinary people after exercise. The increased inflammatory response and repetitive muscle damage by exercising can cause injury, negatively impact the length of rehabilitation after an injury, and reduce the ability to exercise $[7,15,16]$. In this study, we analyzed the levels of serum CK and LDH to verify the changes in muscle damage after exercise in the $\mathrm{NN}$ and $\mathrm{HH}$ environments, and establish the recovery effect of HBOT treatment.

The results of this study showed that the serum CK and LDH levels were significantly increased after exercising in all groups, and there was no significant difference between the exercise environments (NN vs. HH). This result implies that treadmill running at an intensity of 75-80\% HRmax can cause muscle damage, and the $\mathrm{HH}$ environment, which was the same as that at an altitude of about $3000 \mathrm{~m}$, did not add additional stress to the exercise-induced muscle damage. Kasai et al. [26] reported that serum myoglobin levels, the index of muscle damage in blood, increased significantly, irrespective of hypoxic or normoxic conditions. Sumi et al. [35] also suggested that endurance exercise in moderately hypoxic conditions $\left(\mathrm{F}_{\mathrm{i}} \mathrm{O}_{2}=14.5 \%\right)$ did not facilitate more exercise-induced muscle damage response or 
cause in endurance athletes than in normoxic conditions $\left(\mathrm{F}_{\mathrm{i}} \mathrm{O}_{2}=20.9 \%\right)$. In addition, the serum $\mathrm{CK}$ levels were significantly decreased in the $\mathrm{HHH}$ group, and the serum $\mathrm{LDH}$ levels were significantly decreased in the $\mathrm{HNN}$ and $\mathrm{HHH}$ groups at the $\mathrm{AE}$ point compared to the $\mathrm{AH}$ point. These results suggested that HBOT treatment in the recovery phase after exercise was effective in mitigating exercise-induced muscle damage. The study by Cervaens et al. [36], which conducted HBOT treatment of rats with muscle injuries, reported that the serum CK levels were significantly decreased and that HBOT was effective for muscle injury recovery. Although some previous studies $[17,37,38]$ conducted in humans reported that HBOT treatment after exercise did not affect muscle damage (injury) or muscular pain relief, additional studies with larger numbers of subjects should be conducted to verify the effect of HBOT.

The limitations of this study were that (1) the number of subjects was small as this is a pilot study, and (2) serum CK and LDH, the indices of muscle damage, tend to continuously increase up to $72 \mathrm{~h}$ after exercise. Therefore, further verification in additional studies is necessary, taking into account the half-life of CK and LDH.

\section{Conclusions}

In conclusion, acute exercise in the NN and $\mathrm{HH}$ environments caused a temporary inflammatory response and muscle damage, but there was no difference between the environmental conditions. In contrast, HBOT treatment in the recovery phase had a positive impact on relieving the inflammatory response and muscle damage after exercise.

Author Contributions: Study design: J.W., J.-H.M., and H.-T.R. Study conduct: J.W., J.-H.M., Y.-H.L., and H.-T.R. Data collection: J.W. and H.-T.R. Data analysis: J.-H.M., Y.-H.L., and H.-T.R. Data interpretation: J.W. and H.-T.R. Drafting manuscript: J.W., J.-H.M., Y.-H.L., and H.-T.R. Revising the manuscript content: J.W., J.-H.M., Y.-H.L., and H.-T.R. All authors have read and agreed to the published version of the manuscript.

Funding: This work was supported by the Dong-A University research fund.

Conflicts of Interest: The authors declare no conflict of interest.

\section{References}

1. Konopka, A.R.; Harber, M.P. Skeletal muscle hypertrophy after aerobic exercise training. Exerc. Sport Sci. Rev. 2014, 42, 53-61. [CrossRef] [PubMed]

2. Pedersen, B.K.; Saltin, B. Exercise as medicine-evidence for prescribing exercise as therapy in 26 different chronic diseases. Scand. J. Med. Sci. Sports 2015, 25, 1-72. [CrossRef] [PubMed]

3. Powers, S.K.; Jackson, M.J. Exercise-induced oxidative stress: Cellular mechanisms and impact on muscle force production. Physiol. Rev. 2008, 88, 1243-1276. [CrossRef] [PubMed]

4. Pal, S.; Chaki, B.; Chattopadhyay, S.; Bandyopadhyay, A. High-Intensity Exercise Induced Oxidative Stress and Skeletal Muscle Damage in Postpubertal Boys and Girls: A Comparative Study. J. Strength Cond. Res. 2018, 32, 1045-1052. [CrossRef]

5. Cheng, A.J.; Jude, B.; Lanner, J.T. Intramuscular mechanisms of overtraining. Redox Biol. 2020, 35, 101480. [CrossRef]

6. Liakos, C.I.; Vyssoulis, G.P.; Michaelides, A.P.; Chatzistamatiou, E.I.; Theodosiades, G.; Toutouza, M.G.; Markou, M.I.; Synetos, A.G.; Kallikazaros, I.E.; Stefanadis, C.I. The effects of angiotensin receptor blockers vs. calcium channel blockers on the acute exercise-induced inflammatory and thrombotic response. Hypertens. Res. 2012, 35, 1193-1200. [CrossRef]

7. Brancaccio, P.; Maffulli, N.; Buonauro, R.; Limongelli, F.M. Serum enzyme monitoring in sports medicine. Clin. Sports Med. 2008, 27,1-18. [CrossRef]

8. Mazzeo, R.S. Physiological responses to exercise at altitude: An update. Sports Med. 2008, 38, 1-8. [CrossRef]

9. Dosek, A.; Ohno, H.; Acs, Z.; Taylor, A.W.; Radak, Z. High altitude and oxidative stress. Respir. Physiol. Neurobiol. 2007, 158, 128-131. [CrossRef]

10. Eltzschig, H.K.; Carmeliet, P. Hypoxia and inflammation. N. Engl. J. Med. 2011, 364, 656-665. [CrossRef] 
11. Santos, S.A.; Silva, E.T.; Caris, A.V.; Lira, F.S.; Tufik, S.; Dos Santos, R.V. Vitamin E supplementation inhibits muscle damage and inflammation after moderate exercise in hypoxia. J. Hum. Nutr. Diet. 2016, 29, 516-522. [CrossRef] [PubMed]

12. Sousa, M.; Teixeira, V.H.; Soares, J. Dietary strategies to recover from exercise-induced muscle damage. Int. J. Food Sci. Nutr. 2014, 65, 151-163. [CrossRef] [PubMed]

13. Jówko, E.; Płaszewski, M.; Cieśliński, M.; Sacewicz, T.; Cieśliński, I.; Jarocka, M. The effect of low level laser irradiation on oxidative stress, muscle damage and function following neuromuscular electrical stimulation. A double blind, randomised, crossover trial. BMC Sports Sci. Med. Rehabil. 2019, 11, 38. [CrossRef] [PubMed]

14. Leeder, J.D.C.; Godfrey, M.; Gibbon, D.; Gaze, D.; Davison, G.W.; Van Someren, K.A.; Howatson, G. Cold water immersion improves recovery of sprint speed following a simulated tournament. Eur. J. Sport Sci. 2019, 19, 1166-1174. [CrossRef]

15. Moghadam, N.; Hieda, M.; Ramey, L.; Levine, B.D.; Guilliod, R. Hyperbaric Oxygen Therapy in Sports Musculoskeletal Injuries. Med. Sci. Sports Exerc. 2020, 52, 1420-1426. [CrossRef]

16. Chen, C.Y.; Chou, W.Y.; Ko, J.Y.; Lee, M.S.; Wu, R.W. Early Recovery of Exercise-Related Muscular Injury by HBOT. Biomed. Res. Int. 2019, 2019, 6289380. [CrossRef]

17. Germain, G.; Delaney, J.; Moore, G.; Lee, P.; Lacroix, V.; Montgomery, D. Effect of hyperbaric oxygen therapy on exercise-induced muscle soreness. Undersea Hyperb. Med. 2003, 30, 135-145.

18. Webster, A.L.; Syrotuik, D.G.; Bell, G.J.; Jones, R.L.; Hanstock, C.C. Effects of hyperbaric oxygen on recovery from exercise-induced muscle damage in humans. Clin. J. Sport Med. 2002, 12, 139-150. [CrossRef]

19. Godman, C.A.; Joshi, R.; Giardina, C.; Perdrizet, G.; Hightower, L.E. Hyperbaric oxygen treatment induces antioxidant gene expression. Ann. N. Y. Acad. Sci. 2010, 1197, 178-183. [CrossRef]

20. Bruce, R.A.; Blackmon, J.R.; Jones, J.W.; Strait, G. Exercising testing in adult normal subjects and cardiac patients. 1963. Ann. Noninvasive Electrocardiol. 2004, 9, 291-303. [CrossRef]

21. Shimoda, M.; Enomoto, M.; Horie, M.; Miyakawa, S.; Yagishita, K. Effects of hyperbaric oxygen on muscle fatigue after maximal intermittent plantar flexion exercise. J. Strength Cond. Res. 2015, 29, 1648-1656. [CrossRef] [PubMed]

22. Hussein, M.H.; Hashimoto, T.; Daoud, G.A.; Kakita, H.; Kato, S.; Goto, T.; Hibi, M.; Kato, T.; Okumura, N.; Tomishige, H.; et al. Oxidative stress after living related liver transplantation subsides with time in pediatric patients. Pediatr. Surg. Int. 2011, 27, 17-22. [CrossRef] [PubMed]

23. Furman, D.; Campisi, J.; Verdin, E.; Carrera-Bastos, P.; Targ, S.; Franceschi, C.; Ferrucci, L.; Gilroy, D.W.; Fasano, A.; Miller, G.W.; et al. Chronic inflammation in the etiology of disease across the life span. Nat. Med. 2019, 25, 1822-1832. [CrossRef] [PubMed]

24. Boukhris, O.; Trabelsi, K.; Abdessalem, R.; Hsouna, H.; Ammar, A.; Glenn, J.M.; Bott, N.; Irandoust, K.; Taheri, M.; Turki, M.; et al. Effects of the 5-m Shuttle Run Test on Markers of Muscle Damage, Inflammation, and Fatigue in Healthy Male Athletes. Int. J. Environ. Res. Public Health 2020, 17, 4375. [CrossRef]

25. Dupuy, O.; Douzi, W.; Theurot, D.; Bosquet, L.; Dugué, B. An Evidence-Based Approach for Choosing Post-exercise Recovery Techniques to Reduce Markers of Muscle Damage, Soreness, Fatigue, and Inflammation: A Systematic Review With Meta-Analysis. Front. Physiol. 2018, 9, 403. [CrossRef]

26. Kasai, N.; Kojima, C.; Sumi, D.; Ikutomo, A.; Goto, K. Inflammatory, Oxidative Stress, and Angiogenic Growth Factor Responses to Repeated-Sprint Exercise in Hypoxia. Front. Physiol. 2019, 10, 844. [CrossRef]

27. Stelzer, I.; Kröpfl, J.M.; Fuchs, R.; Pekovits, K.; Mangge, H.; Raggam, R.B.; Gruber, H.J.; Prüller, F.; Hofmann, P.; Truschnig-Wilders, M.; et al. Ultra-endurance exercise induces stress and inflammation and affects circulating hematopoietic progenitor cell function. Scand. J. Med. Sci. Sports 2015, 25, e442-e450. [CrossRef]

28. Barnett, A. Using recovery modalities between training sessions in elite athletes: Does it help? Sports Med. 2006, 36, 781-796. [CrossRef]

29. Sugita, M.; Kapoor, M.P.; Nishimura, A.; Okubo, T. Influence of green tea catechins on oxidative stress metabolites at rest and during exercise in healthy humans. Nutrition 2016, 32, 321-331. [CrossRef]

30. Martarelli, D.; Pompei, P. Oxidative stress and antioxidant changes during a 24-hours mountain bike endurance exercise in master athletes. J. Sports Med. Phys. Fitness 2009, 49, 122-127.

31. Aoki, K.; Nakao, A.; Adachi, T.; Matsui, Y.; Miyakawa, S. Pilot study: Effects of drinking hydrogen-rich water on muscle fatigue caused by acute exercise in elite athletes. Med. Gas. Res. 2012, 2, 12. [CrossRef] [PubMed]

32. Powers, S.K.; Radak, Z.; Ji, L.L. Exercise-induced oxidative stress: Past, present and future. J. Physiol. 2016, 594, 5081-5092. [CrossRef] [PubMed] 
33. American College of Sports Medicine. ACSM's Guidelines for Exercise Testing and Prescription, 7th ed.; Lippincott Williams \& Wilkins: Philadelphia, PA, USA, 2006.

34. Bachi, A.L.; Sierra, A.P.; Rios, F.J.; Gonçalves, D.A.; Ghorayeb, N.; Abud, R.L.; Victorino, A.B.; Dos Santos, J.M.; Kiss, M.A.; Pithon-Curi, T.C.; et al. Athletes with higher $\mathrm{VO}_{2}$ max present reduced oxLDL after a marathon race. BMJ Open Sport Exerc. Med. 2015, 1, 000014. [CrossRef] [PubMed]

35. Sumi, D.; Kojima, C.; Goto, K. Impact of Endurance Exercise in Hypoxia on Muscle Damage, Inflammatory and Performance Responses. J. Strength Cond. Res. 2018, 32, 1053-1062. [CrossRef] [PubMed]

36. Cervaens Costa Maia, M.; Camacho, O.F.; Pinto Marques, A.F.; Barata de Silva Coelho, P.M. Hyperbaric oxygen therapy treatment for the recovery of muscle injury induced in rats. Diving Hyperb. Med. 2013, 43, 222-225. [PubMed]

37. Branco, B.H.; Fukuda, D.H.; Andreato, L.V.; Santos, J.F.; Esteves, J.V.; Franchini, E. The Effects of Hyperbaric Oxygen Therapy on Post-Training Recovery in Jiu-Jitsu Athletes. PLoS ONE 2016, 11, e0150517. [CrossRef] [PubMed]

38. Harrison, B.C.; Robinson, D.; Davison, B.J.; Foley, B.; Seda, E.; Byrnes, W.C. Treatment of exercise-induced muscle injury via hyperbaric oxygen therapy. Med. Sci. Sports Exerc. 2001, 33, 36-42. [CrossRef]

(C) 2020 by the authors. Licensee MDPI, Basel, Switzerland. This article is an open access article distributed under the terms and conditions of the Creative Commons Attribution (CC BY) license (http://creativecommons.org/licenses/by/4.0/). 